Nucleation rate of colour superconducting droplets in protoneutron stars

This article has been downloaded from IOPscience. Please scroll down to see the full text article.

2013 J. Phys. G: Nucl. Part. Phys. 40035201

(http://iopscience.iop.org/0954-3899/40/3/035201)

View the table of contents for this issue, or go to the journal homepage for more

Download details:

IP Address: 188.135.8.75

The article was downloaded on 02/02/2013 at 05:55

Please note that terms and conditions apply. 


\title{
Nucleation rate of colour superconducting droplets in protoneutron stars
}

\author{
T A S do Carmo ${ }^{1}$, G Lugones ${ }^{1}$ and A G Grunfeld ${ }^{2,3,4}$ \\ ${ }^{1}$ Centro de Ciências Naturais e Humanas, Universidade Federal do ABC, Rua Santa Adélia, 166, \\ 09210-170, Santo André, Brazil \\ 2 Department of Physics, Sultan Qaboos University, PO Box 36 Al-Khode 123 Muscat, \\ Sultanate of Oman \\ ${ }^{3}$ CONICET, Rivadavia 1917, (1033) Buenos Aires, Argentina \\ ${ }^{4}$ Departamento de Física, Comisión Nacional de Energía Atómica, (1429) Buenos Aires, \\ Argentina \\ E-mail: german.lugones@ufabc.edu.br
}

Received 19 October 2012

Published 22 January 2013

Online at stacks.iop.org/JPhysG/40/035201

\begin{abstract}
We analyse the nucleation of quark matter droplets under protoneutron star conditions. We adopt a two-phase framework in which the hadronic phase is described through a nonlinear Walecka model and the just deconfined matter by the MIT bag model including colour superconductivity. Surface tension and curvature energy are calculated self-consistently within the multiple reflection expansion formalism. We impose flavour conservation during the transition, which means that the just deconfined quark droplet is transiently out of equilibrium with respect to weak interactions. Our results show that trapped neutrinos slightly increase the critical density for deconfinement and that colour superconductivity significantly decreases such density at low temperatures. We also show that the nucleation rate is negligible for droplets larger than 100-200 fm and is huge for smaller droplets provided that the temperature is low enough. We compare our results with previous calculations using the Nambu-Jona-Lasinio model with colour superconductivity and the MIT bag model without colour superconductivity. We conclude that the deconfinement transition should be triggered instantaneously when a density slightly larger than the bulk transition density is reached at some layer of a protoneutron star. Since colour superconductivity lowers the transition density at low temperatures, the transition is likely to occur after significant cooling in a massive enough protoneutron star.
\end{abstract}

(Some figures may appear in colour only in the online journal)

\section{Introduction}

Understanding the behaviour of strongly interacting matter for moderate temperatures and high baryonic density is one of the main tasks in astrophysics for describing the interior of 
neutron stars (NS) and regions close to the core of collapsing stars. In the interior of these objects, at high densities, the deconfinement transition from hadronic to quark matter might occur. This transition, in astrophysical compact and dense objects, is basically studied in two scenarios: in protoneutron stars (PNS) and NS. The PNS are compact objects remaining after the gravitational collapse and supernovae explosion of a massive star. Initially the temperature is about a few tens of $\mathrm{MeV}$ and the neutrinos are temporarily trapped in the interior of the PNS. After a few seconds of evolution, the neutrinos are radiated, and the PNS cools down turning into an NS. The interior of these objects can reach a density well above the nuclear saturation density $\left(\rho_{0}\right)$, and in this scenario the hadronic matter can suffer a deconfinement transition into quark matter [1-5]. The phase transition should begin with the nucleation of a small $\left(\sim 10^{-14} \mathrm{~m}\right)$ quark matter droplet near the centre of the star [3, 6-11] that may later grow through a combustion process converting a macroscopic portion of the star into quark matter [12].

Even though hadrons are a bound state of quarks, we cannot describe with a single model the thermodynamics of the transition from hadronic to quark matter. Then, we need to adopt different models to describe each phase. In this paper, we analyse the deconfinement transition in PNS conditions employing the MIT bag model in the description of quark matter. For the hadronic phase, we use a model based on a relativistic Lagrangian of hadrons interacting via the exchange of $\sigma, \rho$ and $\omega$ mesons. The nucleation is treated as a first-order phase transition and the finite size effects of creating a droplet of deconfined quark matter in the hadronic environment are described using the multiple reflection expansion (MRE) framework [13-17]. Two important features we consider in our work are: (a) quark flavour is conserved during the deconfinement transition because it is driven by strong interactions $[6-11]^{5}$ and (b) when colour superconductivity is included together with flavour conservation, the most likely configuration of the just deconfined phase is two-flavor color superconductor (2SC) provided the pairing gap is large enough [7, 13].

The paper is organized as follows. In section 2, we present the main aspects of the nonlinear Walecka model describing the hadronic phase. In section 3, we present the generalities of the model we use for the quark phase and the MRE formalism. In section 4, we study the deconfinement transition at finite temperature for different neutrino trapping conditions. In section 5 , we present our results followed by a summary and conclusions in section 6 .

\section{Hadronic matter}

We use a nonlinear Walecka model for describing the hadronic phase (see e.g. [3] and references therein) composed of the baryon octet $\left(n, p, \Lambda, \Sigma^{+}, \Sigma^{0}, \Sigma^{-}, \Xi^{-}, \Xi^{0}\right)$, electrons $e^{-}$, electron neutrinos $v_{e}$ and the corresponding antiparticles. The Lagrangian for this model is the following [19]:

$$
\begin{aligned}
\mathcal{L}= & \mathcal{L}_{B}+\mathcal{L}_{M}+\mathcal{L}_{L} \\
= & \sum_{B=n, p, \Lambda, \Sigma^{+, 0,-}, \Xi^{-, 0}} \bar{\psi}_{B}\left[\gamma^{\mu}\left(\mathrm{i} \partial_{\mu}-x_{\omega B} g_{\omega} \omega_{\mu}-x_{\rho B} g_{\rho} \vec{\tau} \cdot \vec{\rho}_{\mu}\right)\right. \\
& \left.-\left(m_{B}-x_{\sigma B} g_{\sigma} \sigma\right)\right] \psi_{B}+\frac{1}{2}\left(\partial_{\mu} \sigma \partial^{\mu} \sigma-m_{\sigma}^{2} \sigma^{2}\right)-\frac{b}{3} m_{N}\left(g_{\sigma} \sigma\right)^{3}
\end{aligned}
$$

5 Works that deal with the structure of hybrid PNS [18] consider the hadron-quark interphase as being in equilibrium under weak interactions. This condition is appropriate for such studies but it is not for the present analysis of the deconfinement of quark droplets. 


$$
\begin{aligned}
& -\frac{c}{4}\left(g_{\sigma} \sigma\right)^{4}-\frac{1}{4} \omega_{\mu \nu} \omega^{\mu \nu}+\frac{1}{2} m_{\omega}^{2} \omega_{\mu} \omega^{\mu}-\frac{1}{4} \vec{\rho}_{\mu \nu} \cdot \vec{\rho}^{\mu \nu}+\frac{1}{2} m_{\rho}^{2} \vec{\rho}_{\mu} \cdot \vec{\rho}^{\mu} \\
& +\sum_{L=e, v_{e}} \bar{\psi}_{L}\left[\mathrm{i} \gamma_{\mu} \partial^{\mu}-m_{L}\right] \psi_{L},
\end{aligned}
$$

where $B, M$ and $L$ refer to baryons, mesons and leptons, respectively. Baryons interact through the exchange of mesons $\sigma, \omega$ and $\rho$. The constants $x_{\sigma B}=g_{\sigma B} / g_{\sigma}, x_{\omega B}=g_{\omega B} / g_{\omega}$ and $x_{\rho B}=g_{\rho B} / g_{\rho}$ are the ratios of the coupling constants of the hyperons to the coupling constants of the nucleons. The equation of state (EoS) is obtained from the above Lagrangian by means of a relativistic mean field model treatment (see [3] and references therein for more details). In order to adapt the EoS to the actual conditions prevailing in PNS, the lepton term includes the contribution of electron neutrinos.

The thermodynamic potential for this model is

$$
\begin{aligned}
\Omega_{H}=-\sum_{B, L} \Omega_{i} & -\frac{1}{2}\left(\frac{g_{\omega}}{m_{\omega}}\right)^{2} \rho_{B}^{\prime 2}+\frac{1}{2}\left(\frac{g_{\sigma}}{m_{\sigma}}\right)^{2}\left(g_{\sigma} \sigma\right)^{2}+\frac{1}{3} b m_{n}\left(g_{\sigma} \sigma\right)^{3}+\frac{1}{4} c\left(g_{\sigma} \sigma\right)^{4} \\
& -\frac{1}{2}\left(\frac{g_{\rho}}{m_{\rho}}\right)^{2} \rho_{I_{3}}^{\prime 2} .
\end{aligned}
$$

The weighted isospin density $\rho_{I_{3}}^{\prime}$ and the weighted baryon density $\rho_{B}^{\prime}$ are given by

$$
\begin{aligned}
& \rho_{I_{3}}^{\prime}=\sum_{i=B} x_{\rho i} I_{3 i} n_{i}, \\
& \rho_{B}^{\prime}=\sum_{i=B} x_{\omega i} n_{i},
\end{aligned}
$$

where $I_{3 i}$ is the third component of the isospin of each baryon and $n_{i}$ is the particle number density of each baryon:

$$
n_{i}=\frac{\gamma_{i}}{(2 \pi)^{3}} \int \mathrm{d}^{3} p\left(f_{i}(T)-\bar{f}_{i}(T)\right),
$$

with $\gamma_{i}$ being the degeneracy factor.

The mean field $g_{\sigma} \sigma$ satisfies the equation:

$$
\left(\frac{g_{\sigma}}{m_{\sigma}}\right)^{-2}\left(g_{\sigma} \sigma\right)+b m_{n}\left(g_{\sigma} \sigma\right)^{2}+c\left(g_{\sigma} \sigma\right)^{3}=\sum_{i=B} x_{\sigma i} n_{i}^{s},
$$

where $n_{i}^{s}$ is the scalar density:

$$
n_{i}^{s}=\frac{\gamma_{i}}{(2 \pi)^{3}} \int \mathrm{d}^{3} p \frac{m_{i}^{*}}{\left(p^{2}+m_{i}^{* 2}\right)^{1 / 2}}\left(f_{i}(T)+\bar{f}_{i}(T)\right) .
$$

From the thermodynamic potential, we obtain the total pressure $P$ and the mass-energy density $\rho$ :

$$
\begin{aligned}
P^{H}=\sum_{i=B, L} P_{i} & +\frac{1}{2}\left(\frac{g_{\omega}}{m_{\omega}}\right)^{2} \rho_{B}^{\prime 2}-\frac{1}{2}\left(\frac{g_{\sigma}}{m_{\sigma}}\right)^{-2}\left(g_{\sigma} \sigma\right)^{2}-\frac{1}{3} b m_{n}\left(g_{\sigma} \sigma\right)^{3}-\frac{1}{4} c\left(g_{\sigma} \sigma\right)^{4} \\
& +\frac{1}{2}\left(\frac{g_{\rho}}{m_{\rho}}\right)^{2} \rho_{I_{3}}^{\prime 2}, \\
\rho^{H}=\sum_{i=B, L} \rho_{i} & +\frac{1}{2}\left(\frac{g_{\omega}}{m_{\omega}}\right)^{2} \rho_{B}^{\prime 2}+\frac{1}{2}\left(\frac{g_{\sigma}}{m_{\sigma}}\right)^{-2}\left(g_{\sigma} \sigma\right)^{2}+\frac{1}{3} b m_{n}\left(g_{\sigma} \sigma\right)^{3}+\frac{1}{4} c\left(g_{\sigma} \sigma\right)^{4} \\
& +\frac{1}{2}\left(\frac{g_{\rho}}{m_{\rho}}\right)^{2} \rho_{I_{3}}^{\prime 2} .
\end{aligned}
$$


Here $P_{i}$ and $\rho_{i}$ are the expressions for a Fermi gas of relativistic, non-interacting particles:

$$
\begin{aligned}
P_{i} & =\frac{1}{3} \frac{\gamma_{i}}{(2 \pi)^{3}} \int \mathrm{d}^{3} p \frac{p^{2}}{\left(p^{2}+m_{i}^{* 2}\right)^{1 / 2}}\left(f_{i}(T)+\bar{f}_{i}(T)\right), \\
\rho_{i} & =\frac{\gamma_{i}}{(2 \pi)^{3}} \int \mathrm{d}^{3} p\left(p^{2}+m_{i}^{* 2}\right)^{1 / 2}\left(f_{i}(T)+\bar{f}_{i}(T)\right),
\end{aligned}
$$

where $f_{i}(T)$ and $\bar{f}_{i}(T)$ are the Fermi-Dirac distribution functions for particles and antiparticles, respectively:

$$
\begin{aligned}
& f_{i}(T)=\left(\exp \left(\left[\left(p^{2}+m_{i}^{* 2}\right)^{1 / 2}-\mu_{i}^{*}\right] / T\right)+1\right)^{-1} \\
& \bar{f}_{i}(T)=\left(\exp \left(\left[\left(p^{2}+m_{i}^{* 2}\right)^{1 / 2}+\mu_{i}^{*}\right] / T\right)+1\right)^{-1} .
\end{aligned}
$$

Note that for baryons we use, instead of masses $m_{i}$ and chemical potentials $\mu_{i}$, 'effective' masses $m_{i}^{*}$ and chemical potentials $\mu_{i}^{*}$ given by

$$
\begin{aligned}
& m_{i}^{*}=m_{i}-x_{\sigma i}\left(g_{\sigma} \sigma\right), \\
& \mu_{i}^{*}=\mu_{i}-x_{\omega i}\left(\frac{g_{\omega}}{m_{\omega}}\right)^{2} \rho_{B}^{\prime}-x_{\rho i} I_{3 i}\left(\frac{g_{\rho}}{m_{\rho}}\right)^{2} \rho_{I_{3}}^{\prime} .
\end{aligned}
$$

The hadronic phase is assumed to be charge neutral and in chemical equilibrium under weak interactions. Electric charge neutrality states:

$$
n_{p}+n_{\Sigma^{+}}-n_{\Sigma^{-}}-n_{\Xi^{-}}-n_{e}=0 .
$$

Chemical weak equilibrium in the presence of trapped electron neutrinos implies that the chemical potential $\mu_{i}$ of each baryon in the hadronic phase is given by

$$
\mu_{i}=q_{B} \mu_{n}-q_{e}\left(\mu_{e}-\mu_{v_{e}}\right),
$$

where $q_{B}$ is its baryon charge and $q_{e}$ is its electric charge. For simplicity, we are assuming that muon and tau neutrinos are not present in the system, and their chemical potentials are set to zero.

The values of the five constants of the model are determined by the properties of nuclear matter. Three of them determine the nucleon couplings to the scalar, vector and vector-isovector mesons $g_{\sigma} / m_{\sigma}, g_{\omega} / m_{\omega}, g_{\rho} / m_{\rho}$, and the other two determine the scalar selfinteractions $b$ and $c$. Moreover, we consider that all hyperons in the octet have the same coupling as the $\Lambda$, and that the coupling of the $\Lambda$ is $90 \%$ of that of the nucleons. Therefore, we have that $x_{\sigma B}, x_{\omega B}$ and $x_{\rho B}$ are equal to 1 for the nucleons and 0.9 for hyperons. In this paper, we use the parametrization labelled as GM4 in [13] with the following values: $\left(g_{\sigma} / m_{\sigma}\right)^{2}=$ $11.79 \mathrm{fm}^{2},\left(g_{\omega} / m_{\omega}\right)^{2}=7.149 \mathrm{fm}^{2},\left(g_{\rho} / m_{\rho}\right)^{2}=4.411 \mathrm{fm}^{2}, b=0.002947, c=-0.001070$. With this parametrization, the EoS is stiff and gives a maximum mass of $2.0 M_{\odot}$ for compact stars.

All the above equations can be solved numerically by specifying three thermodynamic quantities, e.g. the temperature $T$, the mass-energy density $\rho^{H}$ and the chemical potential of electron neutrinos in the hadronic phase $\mu_{v_{e}}^{H}$.

\section{Quark matter}

\subsection{Formalism for quark matter in bulk}

The quark phase is composed of $u, d$ and $s$ quarks, electrons, electron neutrinos and the corresponding antiparticles. We describe this phase using the MIT bag model at 
finite temperature with zero strong coupling constant, $m_{u, d}=0$ and strange quark mass $m_{s}=150 \mathrm{MeV}$. The total thermodynamic potential for the quark matter phase can be written as

$$
\Omega_{Q}=\sum_{c, f} \Omega_{c f}+\sum_{L} \Omega_{L}+B
$$

where $f=u, d, s$ is the flavour index, $c=r, g, b$ is the colour index and $L$ stands for the leptons. The contribution for free unpaired quarks is given by

$$
\Omega_{c f}=-\frac{\gamma T}{2 \pi^{2}} \int_{0}^{\infty} k^{2} \ln \left[1+\mathrm{e}^{-\left(\frac{E_{c f}-\mu_{c f}}{T}\right)}\right] \mathrm{d} k
$$

with $E_{c f}=\sqrt{k^{2}+m_{c f}^{2}}$ being the particle energy and $\mu_{c f}$ the chemical potential. In the case of paired quarks, we consider [20]

$$
\Omega_{c f}=-\frac{\gamma T}{2 \pi^{2}} \int_{0}^{\infty} k^{2} \ln \left[1+\mathrm{e}^{-\frac{\varepsilon_{c f}}{T}}\right] \mathrm{d} k
$$

where $\varepsilon_{c f}= \pm \sqrt{\left(E_{c f}-\mu_{c f}\right)^{2}+\Delta^{2}}$ is the single-particle energy dispersion relation when it acquires an energy gap $\Delta$. Note that, for particles, we can obtain equation (19) from equation (20) in the limit $\Delta=0$, by taking the minus sign in the dispersion relation for $E_{c f}<\mu_{c f}$ and the plus sign when $E_{c f}>\mu_{c f}$ (see e.g. [20]).

The gap equations for a colour-superconducting condensate with total spin $J=0$ have been derived perturbatively in dense QCD [21]. To leading order in weak coupling, the temperature dependence of the condensate is identical to that in BCS-like theories [21]. Then, we consider the following temperature dependence for the gap parameter in equation (20):

$$
\Delta(T)=\Delta_{0} \sqrt{1-\left(\frac{T}{T_{c}}\right)^{2}}
$$

where the critical temperature is $T_{c}=0.57 \Delta_{0}$ [21].

Each lepton species contributes with a term of the form

$$
\Omega_{L}=-\frac{\gamma T}{2 \pi^{2}} \int_{0}^{\infty} k^{2} \ln \left[1+\mathrm{e}^{-\left(\frac{E_{L}-\mu_{L}}{T}\right)}\right] \mathrm{d} k,
$$

with $L=e^{-}, e^{+}, v_{e}, \bar{v}_{e}$ and $E_{L}=\sqrt{k^{2}+m_{L}^{2}}$. The degeneracy factor is $\gamma=2,2,1$ for quarks, electrons and neutrinos, respectively. In all cases, the antiparticles contribution is obtained considering $\bar{\mu}=-\mu$.

In the present model, the quantities $B, m_{s}$ and $\Delta_{0}$ are free parameters. According to their values, the energy per baryon of three flavour deconfined matter (composed of quarks $u, d$ and $s$ ) at zero pressure and temperature can be higher or lower than the mass of the neutron $m_{n}$. This condition defines the so-called stability windows $[22,23]$, which are the regions in 
the $m_{s}-B$ parameter space where quark matter is self-bound, i.e. it is the true ground state of strongly interacting matter. In this paper, we use $B=100 \mathrm{MeV} \mathrm{fm}{ }^{-3}$ corresponding to absolutely stable quark matter and $B\left(\mathrm{MeV} \mathrm{fm}^{-3}\right)=160353$ corresponding to quark matter allowed only at high pressures. The case $B=353 \mathrm{MeV} \mathrm{fm}^{-3}$ leads to an EoS rather similar to set 1 of the Nambu-Jona-Lasinio (NJL) model used in $[3,13]$.

The here-considered just deconfined phase is out of chemical equilibrium with respect to weak interactions. As we will see in the following section, the chemical potentials $\mu_{c f}$, $\mu_{e}$ and $\mu_{v_{e}}$ are related among each other through flavour conservation, color neutrality and pairing conditions.

\subsection{Finite size effects}

In order to study the formation of finite size droplets of quark matter, we use the MRE formalism [13-17]. For a finite spherical droplet, the modified density of states is given by

$$
\rho_{\mathrm{MRE}}\left(k, m_{f}, R\right)=1+\frac{6 \pi^{2}}{k R} f_{S}+\frac{12 \pi^{2}}{(k R)^{2}} f_{C}
$$

with

$$
\begin{aligned}
& f_{S}=-\frac{1}{8 \pi}\left[1-\frac{2}{\pi} \arctan \left(\frac{k}{m_{f}}\right)\right], \\
& f_{C}=\frac{1}{12 \pi^{2}}\left[1-\frac{3 k}{2 m_{f}}\left(\frac{\pi}{2}-\arctan \left(\frac{k}{m_{f}}\right)\right)\right] .
\end{aligned}
$$

The $f_{S}$ and $f_{C}$ terms correspond to the contributions of surface and curvature, respectively, and the drop radius is given by $R$. As in [13], we use the Madsen ansatz [15] to take into account the finite quark mass contributions.

The MRE formalism is included in the EoS by means of the following replacement in the thermodynamic integrals:

$$
\int_{0}^{\infty} \mathrm{d}^{3} k \rightarrow \int_{\Lambda_{\mathrm{IR}}}^{\infty} \mathrm{d}^{3} k \rho_{\mathrm{MRE}}
$$

According to $[16,17]$, the density of states for massive quarks is reduced if compared with the bulk density, leading to a negative density of states for a range of small momentum. In order to avoid this nonphysical effect, one can introduce an infrared cutoff $\Lambda_{\mathrm{IR}}$ in momentum space $k[16,17]$. For calculating the value of $\Lambda_{\mathrm{IR}}$, we need to solve the equation $\rho_{\mathrm{MRE}}=0$ with respect to the momentum $k$ and take the larger root as the IR cutoff. Then, this cutoff depends as well on the radius of the spherical drop and the quark mass.

Performing the above replacement into equation (19), the thermodynamic potential for the quark matter phase reads

$$
\Omega_{Q}^{\mathrm{MRE}}=-P^{Q} V^{Q}+\sigma A+\zeta C
$$

Here, $A=4 \pi R^{2}$ is the spherical drop area, $C=8 \pi R$ the curvature and $V^{Q}=\frac{4}{3} \pi R^{3}$ the volume. The pressure $P^{Q}$ is given by

$$
P^{Q} \equiv-\left.\frac{\partial \Omega_{Q}^{\mathrm{MRE}}}{\partial V^{Q}}\right|_{T, \mu, A, C}
$$


the surface tension is

$$
\left.\sigma \equiv \frac{\partial \Omega_{Q}^{\mathrm{MRE}}}{\partial A}\right|_{T, \mu, V^{Q}, C}
$$

and the curvature energy density is obtained as follows:

$$
\left.\zeta \equiv \frac{\partial \Omega_{Q}^{\mathrm{MRE}}}{\partial C}\right|_{T, \mu, A, V^{Q}}
$$

\section{Nucleation of quark droplets in hadronic matter}

To calculate the conditions for the transition we assume thermal, chemical and mechanical equilibrium between the quark droplet and its hadronic environment. Thermal and chemical equilibrium means that the temperature $T$ and the Gibbs free energy per baryon $g$ are equal in both the hadronic $(\mathrm{H})$ and the quark matter $(\mathrm{Q})$ phases:

$$
\begin{aligned}
& T^{H}=T^{Q} \equiv T, \\
& g^{H}\left(T, \rho^{H}, \mu_{v_{e}}^{H}\right)=g^{Q}\left(T,\left\{\mu_{f c}\right\}, \mu_{e}^{Q}, \mu_{v_{e}}^{Q}\right) .
\end{aligned}
$$

To obtain the condition for mechanical equilibrium, we write the total thermodynamic potential for the combination of a just nucleated quark matter drop immersed in a homogeneous hadronic environment: $\Omega=\Omega_{H}+\Omega_{Q}^{\mathrm{MRE}}$, where $\Omega_{H}=-P^{H} V^{H}$ for the hadronic phase. The condition for mechanical equilibrium is obtained from $\partial \Omega / \partial R=0$ and reads [13, 24]:

$P^{Q}\left(T,\left\{\mu_{f c}\right\}, \mu_{e}^{Q}, \mu_{v_{e}}^{Q}\right)-P^{H}\left(T, \rho^{H}, \mu_{v_{e}}^{H}\right)=\frac{2}{R} \sigma\left(T,\left\{\mu_{f c}\right\}, \mu_{e}^{Q}, \mu_{v_{e}}^{Q}\right)+\frac{2}{R^{2}} \zeta\left(T,\left\{\mu_{f c}\right\}, \mu_{e}^{Q}, \mu_{v_{e}}^{Q}\right)$.

On the other hand, deconfinement is driven by strong interactions and therefore quark and lepton flavours must be conserved during the deconfinement transition [6-11]. When a small quark matter drop is nucleated at the core of a compact star, the abundances of all particle species inside it must be initially the same as in the hadronic matter from which it has been originated. Thus, we have

$$
Y_{f}^{H}\left(T, \rho^{H}, \mu_{v_{e}}^{H}\right)=Y_{f}^{Q}\left(T,\left\{\mu_{f c}\right\}, \mu_{e}^{Q}, \mu_{v_{e}}^{Q}\right),
$$

with $f=u, d, s, e, v_{e}$, with $Y_{f}^{H} \equiv n_{f}^{H} / n_{B}^{H}$ and $Y_{f}^{Q} \equiv n_{f}^{Q} / n_{B}^{Q}$ being the abundances of each particle species in the hadronic and quark phase respectively. Note that, since the hadronic phase is assumed to be electrically neutral, flavour conservation ensures automatically the charge neutrality of the just deconfined quark phase. As a consequence of equation (34), in this work, the just deconfined phase is out of $\beta$ equilibrium.

Additionally, the deconfined phase must be locally colourless, i.e. it must be composed of an equal number of red, green and blue quarks:

$$
\begin{aligned}
& n_{r}\left(T,\left\{\mu_{f r}\right\}\right)=n_{g}\left(T,\left\{\mu_{f g}\right\}\right), \\
& n_{r}\left(T,\left\{\mu_{f r}\right\}\right)=n_{b}\left(T,\left\{\mu_{f b}\right\}\right) .
\end{aligned}
$$

Finally, it has been shown that when colour superconductivity is included together with flavour conservation and colour neutrality, the most likely configuration of the just deconfined 
phase is 2SC provided the pairing gap is large enough [7]. Thus, in order to allow for pairing between quarks $d_{r}$ with $u_{g}$ and between quarks $u_{r}$ with $d_{g}$ we impose that:

$$
\begin{aligned}
& n_{u r}\left(T, \mu_{u r}\right)=n_{d g}\left(T, \mu_{d g}\right), \\
& n_{d r}\left(T, \mu_{d r}\right)=n_{u g}\left(T, \mu_{u g}\right) .
\end{aligned}
$$

Equations (31)-(38) together with the assumption that $\mu_{s r}=\mu_{s g}=\mu_{s b}$ allow us to determine the transition density for given values of the droplet radius, the temperature and the chemical potential of the neutrinos in the hadronic phase.

The theory of homogeneous nucleation predicts a rate of nucleation of a droplet of radius $R$ as given by $[15,24]$

$$
\Gamma \approx T^{4} \mathrm{e}^{-\Delta \Omega / T}
$$

where $\Delta \Omega$, the free energy involved in the formation of the droplet, is given by

$$
\Delta \Omega=-\frac{4}{3} \pi R^{3}\left(P^{Q}-P^{H}\right)+4 \pi R^{2} \sigma+8 \pi R \zeta .
$$

The prefactor $T^{4}$ is included in equation (39) on dimensional grounds because the nucleation rate is largely dominated by the exponential $[9,13,25,26]$, i.e. we always have $\log _{10} \Gamma \approx \log _{10}$ (prefactor) $-\Delta \Omega /[T \ln (10)]$ with the second term much larger than the first. A more elaborate statistical prefactor has been developed in the literature [27]. However, the determination of such a prefactor involves the specification of transport coefficients such as the thermal conductivity, and the shear and bulk viscosities, which are not well known for ultradense matter (see [1,27] for more details). We have checked that the prefactor given in [1] is in fact very different from the $T^{4}$ factor, but it is not dominant with respect to the exponential for the conditions encountered in our calculations. Thus, our results are not significantly affected by the choice in equation (39). The nucleation time $\tau$ is the typical time needed to nucleate a droplet of radius $R$ and is given by $\tau=\left(\frac{4}{3} \pi R^{3} \Gamma\right)^{-1}$.

\section{Results}

In figure 1, we show the mass-energy density and the temperature at which the conversion of a portion of hadronic matter into a quark droplet is energetically favourable. By comparing left and right panels, we can see that curves with $\mu_{v_{e}}^{H}=200 \mathrm{MeV}$ are displaced to the right with respect to the same curves for $\mu_{v_{e}}^{H}=0 \mathrm{MeV}$. In other words, the trapped neutrinos push the transition density to higher values, i.e. the trapped neutrinos tend to inhibit the transition, although the effect is not very large. This behaviour is also reported in [8]. Another interesting feature of the curves shown in figure 1 is that for $T<T_{c} \approx 57 \mathrm{MeV}$ there is a significant decrease of the transition density, which is associated with the appearance of colour superconductivity below $T_{c}$. These results are in agreement with similar calculations that employ the NJL model for quark matter [13]. As expected, we found that the transition density increases when the drop radius decreases, as a consequence of the larger contribution of the surface and curvature terms in equation (33).

The behaviour of the transition density with the gap parameter $\Delta_{0}$ is shown in figure 2 for different values of $\mu_{v_{e}}^{H}, T$ and $B$. Note that the transition density is a decreasing function of the gap parameter $\Delta_{0}$. The effect is strong, e.g. the transition density for $\Delta_{0} \sim 100 \mathrm{MeV}$ is much smaller than for $\Delta_{0}=0 \mathrm{MeV}$. For sufficiently small $\Delta_{0}$, the transition density has constant values. This is because this part of the curve corresponds to temperatures that are larger than the critical temperature $T_{c}=0.57 \Delta_{0}$, and therefore the pairing gap $\Delta(T)$ is zero. 

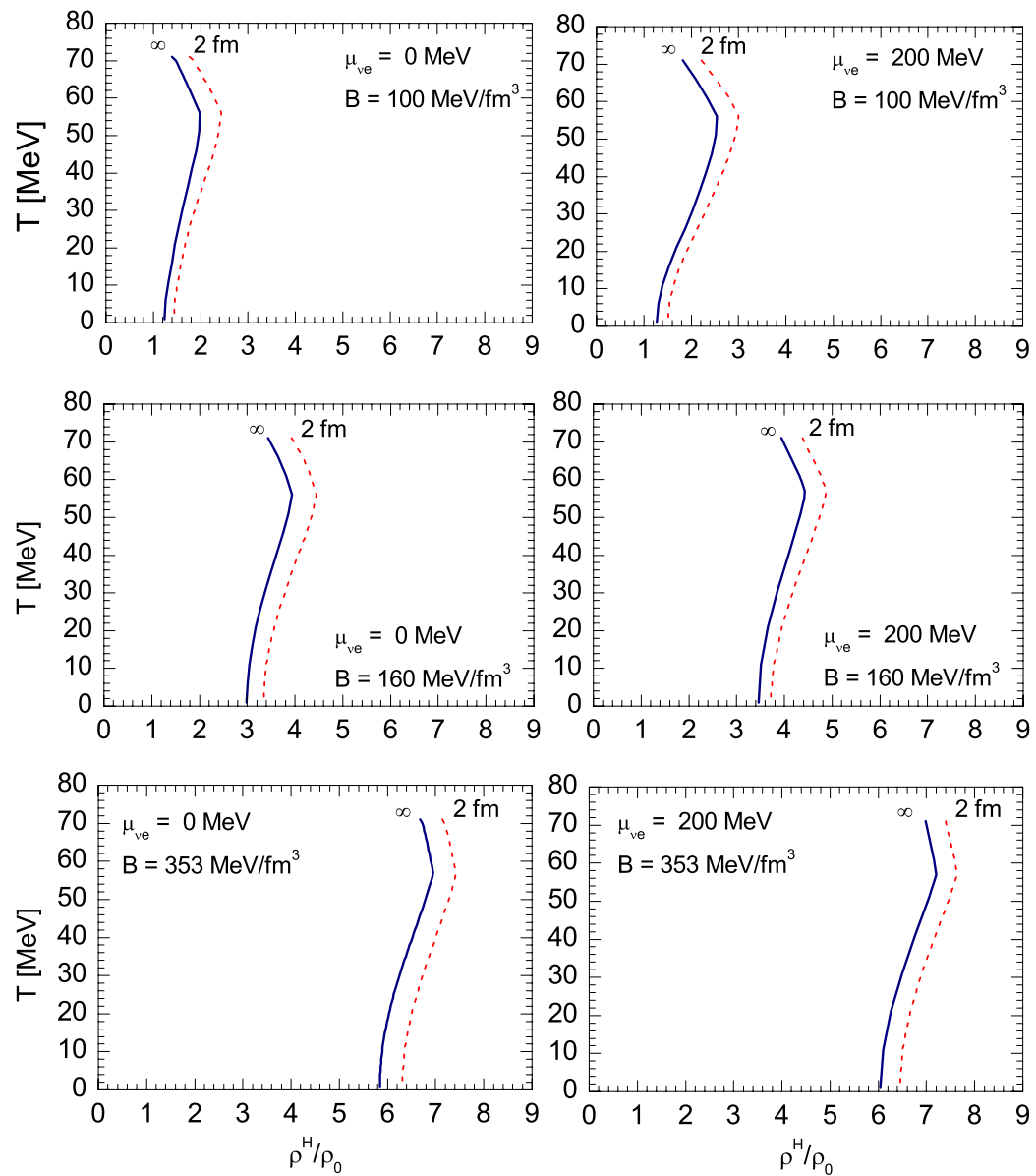

Figure 1. Mass-energy density and temperature of hadronic matter at which deconfinement is energetically favoured for $R=\infty$ and $R=2 \mathrm{fm}$. The deconfinement density for finite size drops is larger than for the bulk case due to surface tension and curvature energy. We have calculated the transition density for several radii and found that for $R \sim 100-200 \mathrm{fm}$ the curves are almost coincident with the case $R=\infty$. The density is given in units of the nuclear saturation density $\rho_{0}$. We use $\Delta_{0}=100 \mathrm{MeV}$ and $B\left(\mathrm{MeV} \mathrm{fm}^{-3}\right)=100,160$ and 353 . We considered different chemical potentials for trapped electron neutrinos in hadron matter, but for simplicity we show only the results for $\mu_{v_{e}}^{H}(\mathrm{MeV})=0$ and 200 .
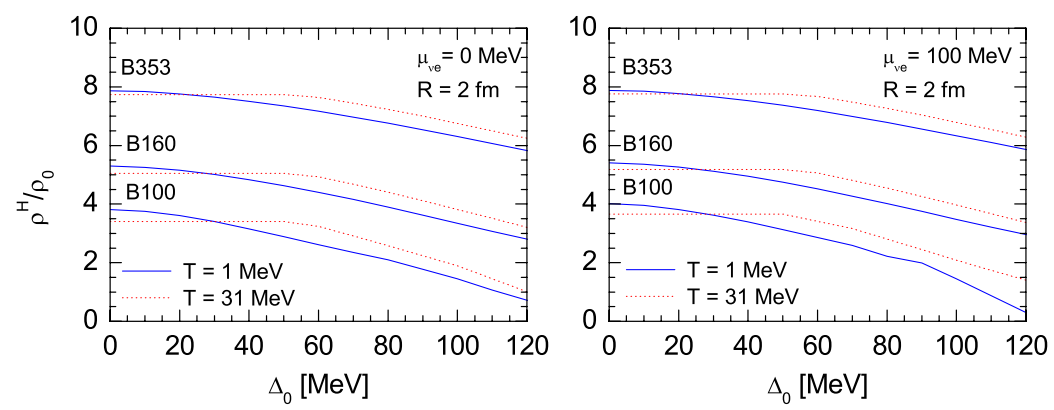

Figure 2. Mass-energy density at which deconfinement is energetically favoured as a function of the parameter $\Delta_{0}$. There is a strong decrease of the transition density $\rho^{H} / \rho_{0}$ for large enough $\Delta_{0}$. 

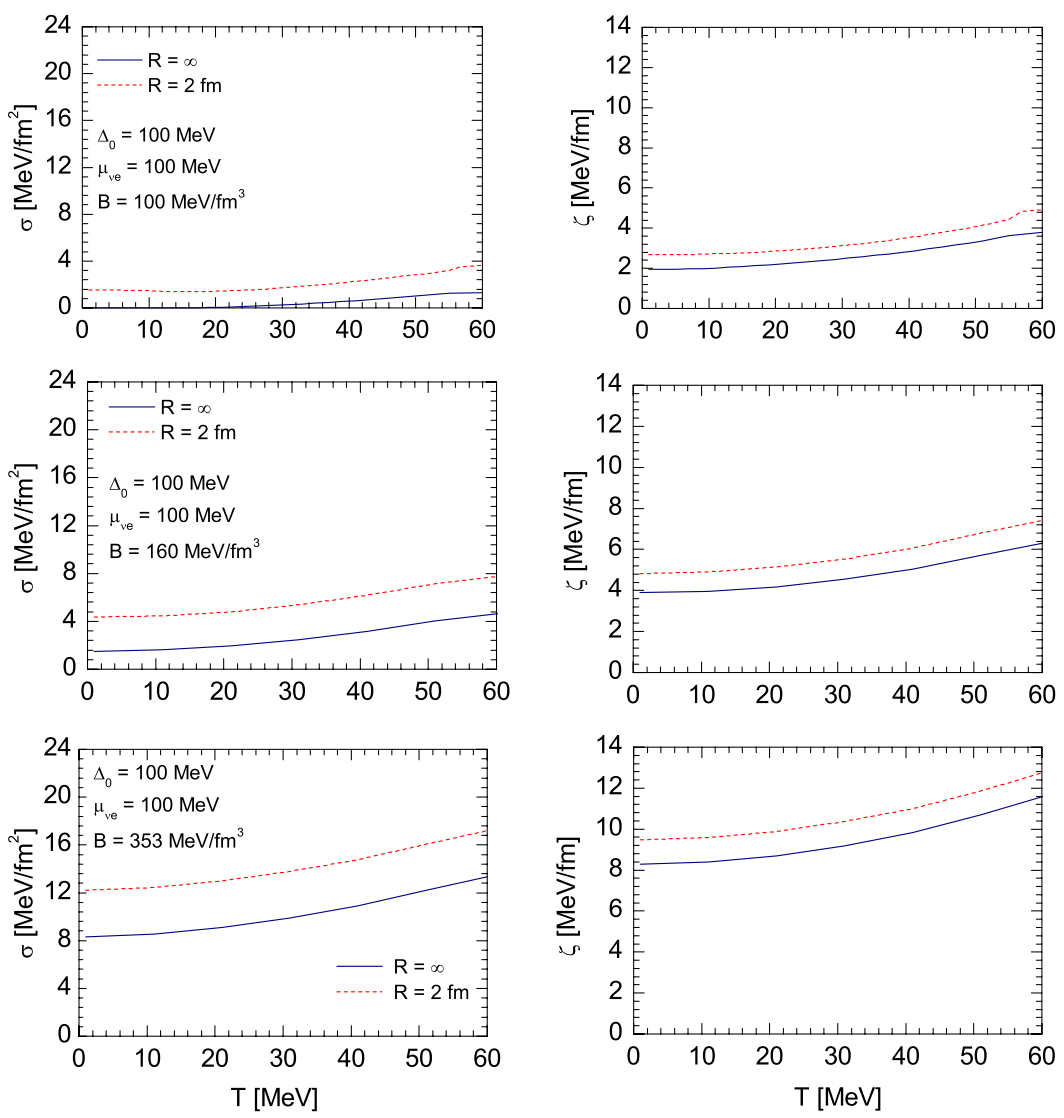

Figure 3. Surface tension $\sigma$ and curvature energy $\zeta$ for droplets with $R=2 \mathrm{fm}$ and $R=\infty$. The curves were calculated using the set of values of the thermodynamic variables that arise from equations (31) and (38), i.e. the same set of values that led to figure 1.

As can be seen in figure 3, we obtain $\sigma \lesssim 18 \mathrm{MeV} \mathrm{fm}^{-2}$ and $\zeta \sim 4-12 \mathrm{MeV} \mathrm{fm}^{-1}$, which are ordinarily an order of magnitude smaller than the values obtained within the NJL model [13]. Our values for $\sigma$ are also smaller than the constant value $\sigma=30 \mathrm{MeV} \mathrm{fm}^{-2}$ assumed in [1].

In figures 4 and 5, we present the results for the nucleation rate given by equation (39). From figure 4 , we see that there are large variations in the value of $\log _{10}\left(\Gamma\left[\mathrm{cm}^{-3} \mathrm{~s}^{-1}\right]\right)$ for different values of $B$, but the qualitative behaviour is nearly the same in all cases: (i) the nucleation rate increases hugely when the temperature falls from 61 to $1 \mathrm{MeV}$, and (ii) the nucleation of droplets larger than some value $R^{*}$ is strongly suppressed (typically $R^{*} \sim 100-200 \mathrm{fm}$ ). In contrast, droplets with $R \lesssim R^{*}$ have a huge nucleation rate at low enough $T$, and in practice should nucleate instantaneously when the bulk transition density is attained at the core of a PNS. In figure 5, we show that the main conclusions obtained from figure 4 are valid for different values of $\Delta_{0}$. Specifically, we note on the right panel that $\log _{10} \Gamma$ is a large negative number for radii above $R^{*}$. On the left panel, we see that $\log _{10} \Gamma$ is a large positive number for radii below $R^{*}$, with the possible exception of some very small drops with $R \lesssim 10 \mathrm{fm}$ 

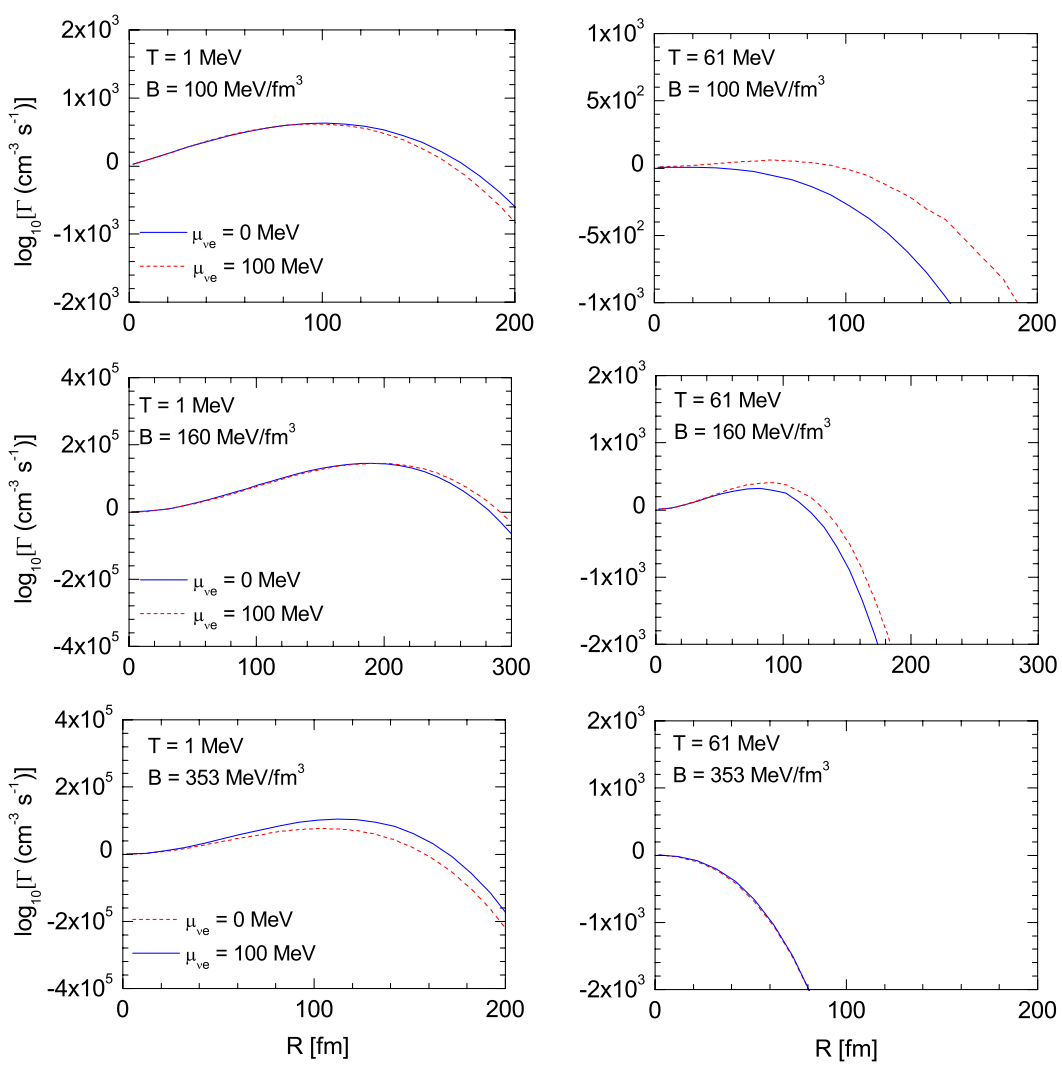

Figure 4. Nucleation rate of droplets as a function of their radii, for $\Delta_{0}=100 \mathrm{MeV}, B$ $\left(\mathrm{MeV} \mathrm{fm}^{-3}\right)=100,160,353, \mu_{v_{e}}^{H}(\mathrm{MeV})=0,100$ and $T(\mathrm{MeV})=1,61$.
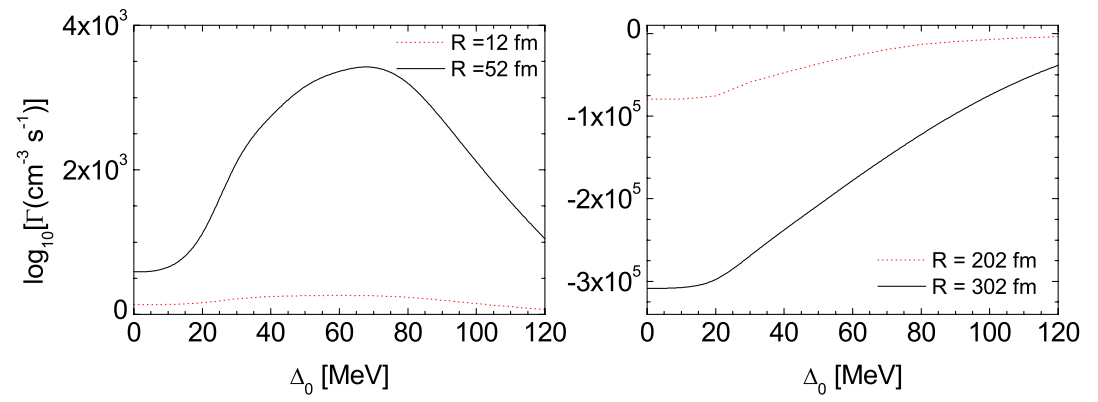

Figure 5. Nucleation rate of droplets as a function of $\Delta_{0}$ for $B\left(\mathrm{MeV} \mathrm{fm}^{-3}\right)=160, \mu_{v_{e}}^{H}(\mathrm{MeV})=$ 100 and $T(\mathrm{MeV})=11$

\section{Summary and conclusions}

In this work, we analyse the nucleation of quark droplets inside a PNS focusing our analysis on the colour superconductivity, trapped neutrinos and finite size effects. To describe strongly interacting matter we consider a different model for each phase. For the hadronic phase, we use the Walecka model including the baryonic octet, electrons and electron neutrinos in equilibrium under weak interactions. The just-deconfined quark matter is described using the MIT bag 
model (considering $u, d$ and $s$ flavours, electrons and electron neutrinos). To determine the energy density at which the deconfinement occurs, we assume a first-order phase transition, impose flavour conservation during the transition and consider the deconfined phase to be in the energetically favoured 2SC state. The effects of finite size of the nucleated droplet are included through the MRE formalism, i.e. the surface tension and the curvature energy are not treated as free parameters, but are calculated self-consistently as a function of the thermodynamic state of the system.

Firstly, we determine the transition density for given values of the droplet radius, the gap parameter $\Delta_{0}$, the temperature and the chemical potential of the neutrinos in the hadronic phase (see figures 1 and 2). Due to the effect of colour superconductivity, there is a significant decrease of the transition density at low temperatures. In most cases, our values for the surface tension (see figure 3 ) turn out to be a factor $\sim 2-10$ smaller that the constant value assumed in [1] within the MIT bag model and between one and two orders of magnitude smaller than the value calculated in [13] using the MRE together with the NJL model. Secondly, we calculate the nucleation rate of quark droplets as a function of their radii for different temperatures, pairing gaps $\Delta_{0}$ and trapped neutrino abundances (see figures 4 and 5). Large droplets have a low transition density because surface and curvature effects tend to be small; but at the same time, their nucleation probability is suppressed because there is a large free energy involved in their formation (due to their large volume). As a consequence, the nucleation rate $\Gamma$ is negligible for droplets larger than $R^{*} \sim 100-200 \mathrm{fm}$. We also show that $\Gamma$ is huge for most radii below $R^{*}$ provided that the temperature is low enough.

All these results are in qualitative agreement with previous calculations obtained within the frame of the NJL model [13]. Quantitatively, the here obtained nucleation rate is very different to the one obtained within the NJL model; nonetheless, both are negligible above some radius $R^{*}$ and huge below it. Moreover, in spite of the large differences in $\sigma, \zeta$ and $\Gamma$ for the MIT and the NJL models, the radius $R^{*}$ is within the same order of magnitude in both cases (some hundreds of fm). Since the transition density for $R \sim 100-200 \mathrm{fm}$ is almost coincident with the bulk transition density and the nucleation rate of such droplets is huge, the deconfinement transition should be triggered instantaneously when a density slightly larger than the bulk transition density shown in figure 1 is reached at some layer of a PNS. Since colour superconductivity lowers the transition density at low temperatures, the transition is likely to occur after significant cooling in a massive enough PNS.

\section{Acknowledgments}

TASdC acknowledges the financial support received from CAPES. GL acknowledges the financial support received from FAPESP.

\section{References}

[1] Bombaci I, Logoteta D, Panda P K, Providencia C and Vidana I 2009 Phys. Lett. B 680448

[2] Mintz B W, Fraga E S, Pagliara G and Schaffner-Bielich J 2010 Phys. Rev. D 81123012

[3] Lugones G, do Carmo T A S, Grunfeld A G and Scoccola N N 2010 Phys. Rev. D 81085012

[4] Lugones G, Grunfeld A G, Scoccola N N and Villavicencio C 2009 Phys. Rev. D 80045017

[5] Hempel M, Pagliara G and Schaffner-Bielich J 2009 Phys. Rev. D 80125014

[6] Iida K and Sato K 1997 Prog. Theor. Phys. 98277

[7] Lugones G and Bombaci I 2005 Phys. Rev. D 72065021

[8] Lugones G and Benvenuto O G 1998 Phys. Rev. D 58083001

Benvenuto O G and Lugones G 1999 Mon. Not. R. Astron. Soc. 304 L25

[9] Olesen M L and Madsen J 1994 Phys. Rev. D 492698 
[10] Bombaci I, Parenti I and Vidaña I 2004 Astrophys. J. 614314

[11] Bombaci I, Lugones G and Vidaña I 2007 Astron. Astrophys. 4621017

[12] Lugones G, Benvenuto O G and Vucetich H 1994 Phys. Rev. D 506100

[13] Lugones G and Grunfeld A G 2011 Phys. Rev. D 84085003

[14] Balian R and Bloch C 1970 Ann. Phys. 60401

[15] Madsen J 1994 Phys. Rev. D 503328

[16] Kiriyama O and Hosaka A 2003 Phys. Rev. D 67085010

[17] Kiriyama O 2005 Phys. Rev. D 72054009

[18] Nicotra O E et al 2006 Phys. Rev. D 74123001

Burgio G F et al 2008 Phys. Rev. D 77085022

Yasutake N et al 2011 Phys. At. Nucl. 741534

Chen $\mathrm{H}$ et al 2012 Phys. Rev. D 86045006

[19] Glendenning N K and Moszkowski S A 1991 Phys. Rev. Lett. 672414

[20] Schmitt A 2010 Dense Matter in Compact Stars: A Pedagogical Introduction (Lecture Notes in Physics vol 811) (Berlin: Springer)

[21] Pisarski R D and Rischke D H 1999 Phys. Rev. D 61074017

[22] Farhi E and Jaffe R L 1984 Phys. Rev. D 302379

[23] Lugones G and Horvath J E 2002 Phys. Rev. D 66074017

[24] Landau L D and Lifshitz E M 1980 Statistical Physics vol 5 3rd edn (Oxford: Butterworth-Heinemann)

[25] Horvath J E, Benvenuto O G and Vucetich H 1992 Phys. Rev. D 453865

[26] Horvath J E 1994 Phys. Rev. D 495590

[27] Langer J S 1968 Phys. Rev. Lett. 21973

Langer J S 1969 Ann. Phys., NY 54258

Langer J S and Turski L A 1973 Phys. Rev. A 83230

Turski L A and Langer J S 1980 Phys. Rev. A 222189

Csernai L and Kapusta J I 1992 Phys. Rev. D 461379

Venugopalan R and Vischer A P 1994 Phys. Rev. E 495849 